\title{
Association of NFE2L2 Gene Polymorphisms with Risk and Clinical Characteristics of Acute Type A Aortic Dissection in Han Chinese Population
}

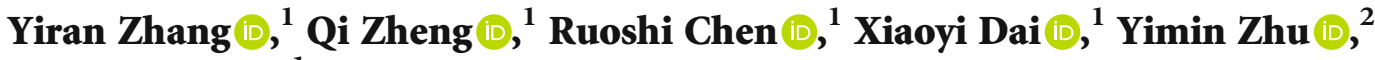 \\ and Liang Ma ${ }^{1}{ }^{1}$ \\ ${ }^{1}$ Department of Cardiovascular Surgery, First Affiliated Hospital, School of Medicine, Zhejiang University, Hangzhou 310003, China \\ ${ }^{2}$ Department of Epidemiology \& Biostatistics, School of Public Health, Zhejiang University, Hangzhou 310058, China
}

Correspondence should be addressed to Yimin Zhu; zhuym@zju.edu.cn and Liang Ma; ml1402@zju.edu.cn

Received 11 May 2021; Revised 15 June 2021; Accepted 1 July 2021; Published 19 July 2021

Academic Editor: Ding-Sheng Jiang

Copyright (c) 2021 Yiran Zhang et al. This is an open access article distributed under the Creative Commons Attribution License, which permits unrestricted use, distribution, and reproduction in any medium, provided the original work is properly cited.

\begin{abstract}
The present study is aimed at investigating the association of NFE2L2 gene polymorphisms with risk and clinical characteristics of acute type A aortic dissection (AAAD) in a Han Chinese population. Six SNPs (rs1806649, rs13001694, rs2364723, rs35652124, rs6721961, and rs2706110) in NFE2L2 were genotyped using SNaPshot Multiplex Kit in 94 adult patients diagnosed with AAAD at our hospital, and 208 healthy Han Chinese subjects from the 1000 Genomes Project were served as the control group. The CC genotype of rs2364723 (CC versus (GC+GG), OR $=2.069,95 \% \mathrm{CI}: 1.222-3.502, p=0.006$ ) and CC genotype of rs35652124 (CC versus (CT+TT), OR $=1.889,95 \% \mathrm{CI}: 1.112-3.210, p=0.018$ ) were identified as risk factors for AAAD. Multivariable linear regression analysis revealed that the CC genotype of $\operatorname{rs} 2364723$ ( $\beta=5.031,95 \%$ CI: $1.878-8.183, p=0.002)$ and CC genotype of rs35652124 ( $\beta=4.751,95 \%$ CI: 1.544-7.958, $p=0.004)$ were associated with increased maximum ascending aorta diameter of AAAD. Patients carrying rs 2364723 CC genotype had a higher incidence of coronary artery involvement (31\% vs. $12 \%, p=0.027)$, while patients carrying rs 35652124 CC genotype had a higher incidence of brain ischemia $(9 \%$ vs. $0 \%, p=0.045)$. In conclusion, NFE2L2 gene polymorphisms were correlated with risk and severity of AAAD in Han Chinese population.
\end{abstract}

\section{Introduction}

Acute type A aortic dissection (AAAD) is a medical emergency with $48.6 \%$ of the patients died before hospital admission [1] and in-hospital mortality rate as high as 32.5\% [2]. Therefore, it is important to identify the pathogenesis and risk factors of AAAD, which could help prevention and early intervention of the disease.

Genetic susceptibility is an important risk factor for AAAD. It has been shown that nearly $20 \%$ AAAD patients also had hereditary disorders such as Marfan syndrome, Loeys-Dietz syndrome, and Turner syndrome [3]. Several recent studies reported that polymorphisms in genes such as TLR4 [4], ALDH2 [5], and Mfn2 [6] were associated with risk of sporadic aortic dissection. However, the genetic determinants of AAAD remained largely undiscovered.
It has been indicated that oxidative stress participated in degeneration and necrosis of aortic media, which played an essential role in the development of aortic dissection. A proteomic study showed that the expression and activity of superoxide dismutase were decreased in the aortic media of patients with thoracic aortic dissection, while the level of lipid peroxidation was increased [7]. It has been shown that increased fluid shear stress on aorta wall led to accumulation of reactive oxygen species (ROS), inducing phenotype switch of vascular smooth muscle cells, which played a vital role in formation of aortic aneurysm and dissection $[8,9]$.

Nuclear factor erythroid 2 like 2 (NFE2L2) is a gene localized on chromosome $2 \mathrm{q} 31.2$, which encodes a transcription factor Nrf2. Nrf2 regulates a number of antioxidative genes by binding to antioxidant response elements (ARE) in their promoters, and the Nrf2-ARE signaling played a protective 
role in various kinds of oxidative stress injury [10]. A number of studies suggested that activating Nrf2-ARE signaling could alleviate apoptosis [11], calcification [12], and phenotype switch [13] of vascular smooth muscle cells. Moreover, several single-nucleotide polymorphisms (SNPs) in NFE2L2 gene have been reported to be associated with coronary artery disease [14], vascular stiffness [15], and cardiovascular mortality [16] in different populations. Thus, we inferred that NFE2L2 gene polymorphisms may be associated with AAAD, while no study has examined this relationship. The present study investigated the association of NFE2L2 gene polymorphisms with risk and clinical characteristics of AAAD in a Han Chinese population.

\section{Materials and Methods}

2.1. Study Population. This study was approved by the ethics committee of the First Affiliated Hospital, School of Medicine, Zhejiang University in China (reference number: IIT2020-277). All participants gave informed consents before inclusion in the study. The inclusion criteria were as follows: (1) Han Chinese patients older than 18 years, (2) aortic dissection diagnosed according to computed tomography angiography (CTA) and echocardiography, and (3) the time from the onset of the symptom to admission within 14 days. The exclusion criteria were as follows: (1) patients with familial aortic dissection or other types of acute aortic syndrome (i.e., intramural hematoma, penetrating ulcer, and iatrogenic/traumatic dissection) and (2) patients who did not sign the informed consents. A total of 94 adult patients diagnosed with AAAD were enrolled in this study between September 2018 and November 2020 at our hospital (Figure 1).

$5 \mathrm{~mL}$ of the peripheral blood was collected from each participant and stored at $-80^{\circ} \mathrm{C}$ for further examination. All blood samples were handled anonymously. Clinical variables of each patient were obtained through review of medical records, which included demographics (age and gender), medical histories (hypertension, diabetes, chronic kidney disease, prior aortic stent implant, prior cardiac surgery, smoking, and bicuspid aortic valve), blood pressure on admission (systolic and diastolic), maximum diameter of ascending aorta (measured by echocardiography preoperatively), severity of aortic valve regurgitation, the site and number of primary/secondary entry tears (according to the operation note), DeBakey classification of the dissection, organ ischemia (brain, coronary artery involvement, and lower limber), and laboratory tests on admission (white blood cell count, platelet count, hemoglobin, high sensitivity C-reactive protein, fibrinogen, $\mathrm{D}$-dimer, serum uric acid, creatine, and urea nitrogen).

The control group consisted of 208 healthy Han Chinese subjects from the publicly available population-based database of the 1000 Genomes Project, which included 103 Han Chinese in Beijing, China (CHB) and 105 Southern Han Chinese, China (CHS) (Figure 1).

2.2. SNP Selection and Genotyping. Six SNPs (rs1806649 [17], rs13001694 [16], rs2364723 [16], rs35652124 [18], rs6721961 $[14,15]$, and rs2706110 [19]) located in NFE2L2 previously reported to be associated with cardiovascular risk were selected for genotyping.

Genomic DNA was extracted from venous blood samples using Trelief Animal Genomic DNA Kit (Beijing TsingKe Biotech Co., Ltd., Beijing, China) according to the manufacturer's instruction. Genotyping of the six SNPs was performed using the SNaPshot Multiplex Kit (Thermo Fisher Scientific, Waltham, MA, USA). Amplification primers and extension primers are listed in Table 1. Briefly, multiplex polymerase chain reaction was performed followed by a single-nucleotide primer extension assay test. The SNPs were detected by capillary electrophoresis using ABI 3730xl DNA Analyzer (Thermo Fisher Scientific, Waltham, MA, USA). The data was analyzed using GeneMapper 4.1 software (Applied Biosystems, Foster City, CA, USA).

2.3. Statistical Analysis. The Hardy-Weinberg equilibrium (HWE) was evaluated using Genetics package in R software to determine the representativeness of the study population. Categorical variables were presented as numbers and proportions and were compared between different groups using Pearson's chi-square or Fisher's exact test, as appropriate. Normally distributed continuous data were presented as the mean \pm SD and were compared using unpaired Student $t$-test. Nonnormally distributed continuous data were presented as median with interquartile range (IQR) and were compared by the Mann-Whitney $U$ test. Dominant, recessive, and homozygote genetic models of inheritance were chosen to evaluate the association between each SNP and AAAD risk with odds ratio (OR) and 95\% confidence interval (95\% CI). Multivariable linear regression model was used to adjust the effect of potential confounders on the association between NFE2L2 gene polymorphisms and maximum ascending aorta diameter. All statistical analyses were processed using SPSS 25.0 software (SPSS Inc., Chicago, IL, USA) and R programming language (version 4.0.0). All $p$ values of $<0.05$ were considered statistically significant.

\section{Results}

3.1. Genotype and Allele Frequencies of NFE2L2 Gene Polymorphisms. The genotype frequencies for all 6 SNPs were in line with HWE in the control group ( $\operatorname{rs1806649}, p=0.379$; rs13001694, $p=0.741 ;$ rs2364723, $p=1.000 ;$ rs35652124, $p=1.000 ;$ rs6721961, $p=0.600 ;$ and rs2706110, $p=0.450)$ and the AAAD group (rs1806649, $p=1.000$; rs13001694, $p=1.000 ; \quad \mathrm{rs} 2364723, p=0.292 ; \quad \mathrm{rs} 35652124, p=0.285$; rs6721961, $p=0.205$; and rs2706110, $p=0.754)$, indicating that the results of the present study have a representative genetic group. The genotype distribution and allele frequency of each SNP are shown in Tables 2 and 3. The genotype distribution of rs2364723 differed significantly between the AAAD group and control group $(p=0.021)$. The allele frequencies of rs2364723 $(p=0.009)$ and $\operatorname{rs35652124}(p=0.021)$ were significantly linked to AAAD risk.

3.2. Association between NFE2L2 Gene Polymorphisms and $A A A D$ Risk. The association between NFE2L2 gene polymorphisms and AAAD risk in different genetic models is shown 


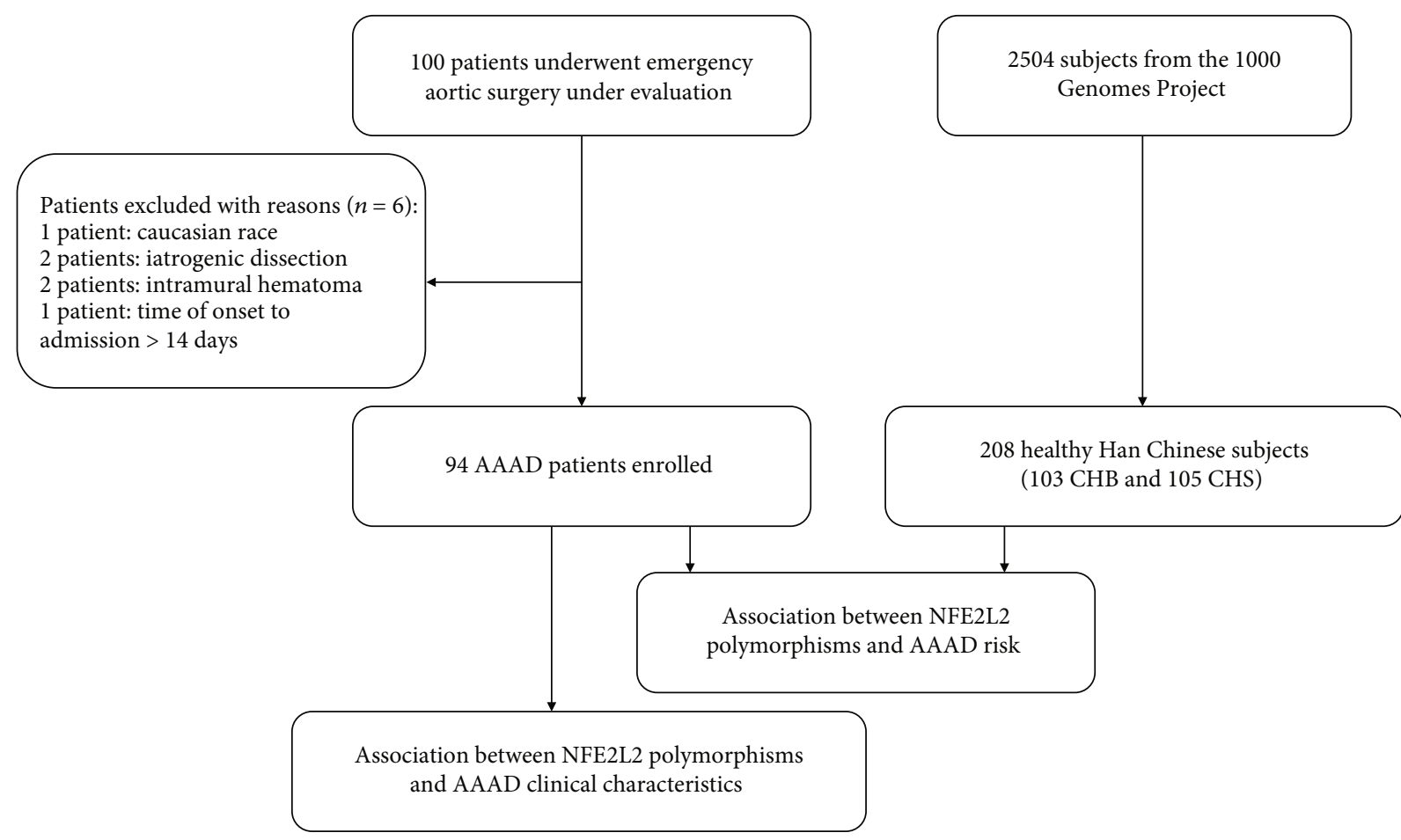

FIgure 1: Flow chart of the study design. AAAD: acute type A aortic dissection; CHB: Han Chinese in Beijing, China; CHS: southern Han Chinese, China.

TABLE 1: Amplification primers and extension primers of selected SNPs.

\begin{tabular}{lcc}
\hline SNPs & \multicolumn{1}{c}{ Amplification primers } & Extension primers \\
\hline \multirow{2}{*}{ rs1806649 } & $\begin{array}{c}\text { Forward: ACTGTCACAGCATTGTCATGTATCA } \\
\text { Reverse: TAGGAATCTTTCAGGGCATGAAG }\end{array}$ & TTTTTTTTTTTTTTAGTCTTAGAGGAACTCATATCCTAAG \\
rs13001694 & $\begin{array}{c}\text { Forward: TTGCAGCTGTAGGTCCGAGA } \\
\text { Reverse: TTTTGTAGGGAAGCCTGCCA }\end{array}$ & TTTTTTTTTTTTTCTGGACAAGTCACTCTACCTGC \\
& Forward: AGCAATTATGTCTGGCACCC & TTTTTTTTTTTTTTTTTTTTTTTGGTTTTGTGTCAATATTTCCTC \\
rs364723 & Reverse: CCAGGAAAAGTTGAAAGCCAAATG & \\
rs652124 & Forward: CTCACTTTACCGCCCGAGAA & TTTTTTTTTTTTTTTTTTTTTTTTTTTTTACACGTGGGAGTTCAGAGG \\
& Reverse: GTTCTCTTGGGGTTCCCGTT & \\
rs2706110 & Forward: CTCACTTTACCGCCCGAGAA & TTTTTTTTTTTTTTTTTTTTTTTTTTTTTTTTTCTAGGGGAGATGTGGACAGC \\
& Reverse: GTTCTCTTGGGGTTCCCGTT & \\
\hline
\end{tabular}

in Table 4. rs2364723 was strongly correlated with the risk of AAAD in the recessive genetic model (CC versus (GC+GG), $\mathrm{OR}=2.069,95 \% \mathrm{CI}: 1.222-3.502, p=0.006)$ and the homozygous model (CC versus GG, OR = 2.333, 95\% CI: 1.177-4.627, $p=0.014$ ), which indicated that the CC genotype of rs 2364723 was a risk factor for AAAD. rs35652124 was strongly correlated with the risk of AAAD in the recessive genetic model (CC versus (CT+TT), OR $=1.889,95 \% \mathrm{CI}: 1.112-3.210, p=0.018)$ and the homozygous model (CC versus TT, OR $=2.125,95 \% \mathrm{CI}$ : $1.076-4.195, p=0.029$ ), which indicated that the CC genotype of rs35652124 was a risk factor for AAAD.

3.3. Association between rs2364723 and rs35652124 Polymorphisms with AAAD Clinical Characteristics. Table 5 summarizes the clinical characteristics of the 94 AAAD patients in different genotype groups. Compared with patients carrying rs2364723 GC+GG genotypes, patients with rs2364723 CC genotype had increased maximum diameter of ascending aorta (median diameter, 49 vs. $42 \mathrm{~mm}, p=0.002$, Table 5 and Figure 2(a)) and higher incidence of coronary artery involvement ( $31 \%$ vs. $12 \%, p=0.027$, Table 5 ). Compared with patients carrying rs35652124 CT+TT genotypes, patients with rs35652124 CC genotype had increased maximum diameter of ascending aorta (median diameter, 49 vs. $43 \mathrm{~mm}, p=0.011$, Table 5 and Figure 2(b)) and higher incidence of brain ischemia ( $9 \%$ vs. $0 \%, p=0.045$, Table 5 ).

3.4. Multivariable Linear Regression Analysis for Maximum Ascending Aorta Diameter. After adjusting for potential confounders including demographics (age and gender) and 
TABLE 2: Genotype distribution of NFE2L2.

\begin{tabular}{|c|c|c|c|c|c|c|}
\hline \multicolumn{2}{|c|}{ SNP } & \multicolumn{3}{|c|}{ Genotype (frequency, \%) } & \multirow[t]{2}{*}{$x^{2}$} & \multirow[t]{2}{*}{$p$ value } \\
\hline \multirow{4}{*}{ rs 1806649} & & TT & CT & $\mathrm{CC}$ & & \\
\hline & Case & $1(1.1)$ & $17(18.1)$ & $76(80.9)$ & \multirow{2}{*}{0.289} & \multirow{2}{*}{0.865} \\
\hline & Control & $3(1.4)$ & $33(15.9)$ & $172(82.7)$ & & \\
\hline & & GG & GA & $\mathrm{AA}$ & & \\
\hline \multirow{2}{*}{ rs13001694 } & Case & $2(2.1)$ & $22(23.4)$ & $70(74.5)$ & \multirow{2}{*}{0.311} & \multirow{2}{*}{0.856} \\
\hline & Control & $4(1.9)$ & $43(20.7)$ & $161(77.4)$ & & \\
\hline \multirow{4}{*}{ rs2364723 } & & $\mathrm{CC}$ & GC & GG & \multirow{4}{*}{7.742} & \multirow{4}{*}{0.021} \\
\hline & Case & $36(38.3)$ & $40(42.6)$ & $18(19.1)$ & & \\
\hline & Control & $48(23.1)$ & $104(50.0)$ & $56(26.9)$ & & \\
\hline & & $\mathrm{CC}$ & CT & $\mathrm{TT}$ & & \\
\hline \multirow{2}{*}{ rs35652124 } & Case & $34(36.2)$ & $41(43.6)$ & $19(20.2)$ & \multirow{2}{*}{5.891} & \multirow{2}{*}{0.053} \\
\hline & Control & $48(23.1)$ & $103(49.5)$ & $57(27.4)$ & & \\
\hline \multirow{4}{*}{ rs6721961 } & & TT & GT & GG & \multirow{4}{*}{4.434} & \multirow{4}{*}{0.109} \\
\hline & Case & $2(2.1)$ & $35(37.2)$ & $57(60.6)$ & & \\
\hline & Control & $15(7.2)$ & $87(41.8)$ & $106(51.0)$ & & \\
\hline & & TT & CT & $\mathrm{CC}$ & & \\
\hline \multirow{2}{*}{ rs 2706110} & Case & $3(3.2)$ & $32(34.0)$ & $59(62.8)$ & \multirow{2}{*}{1.005} & \multirow{2}{*}{0.605} \\
\hline & Control & $10(4.8)$ & $79(38.0)$ & $119(57.2)$ & & \\
\hline
\end{tabular}

TABLE 3: Allele frequency of NFE2L2.

\begin{tabular}{|c|c|c|c|c|c|}
\hline \multicolumn{2}{|c|}{ SNP } & \multicolumn{2}{|c|}{ Allele (frequency, \%) } & \multirow[t]{2}{*}{$\chi^{2}$} & \multirow[t]{2}{*}{$p$ value } \\
\hline \multirow{4}{*}{ rs1806649 } & & $\mathrm{T}$ & $\mathrm{C}$ & & \\
\hline & Case & $19(10.1)$ & $169(89.9)$ & \multirow{2}{*}{0.080} & \multirow{2}{*}{0.778} \\
\hline & Control & $39(9.4)$ & $377(90.6)$ & & \\
\hline & & G & A & & \\
\hline \multirow{2}{*}{ rs13001694 } & Case & $26(13.8)$ & $162(86.2)$ & \multirow{2}{*}{0.287} & \multirow{2}{*}{0.592} \\
\hline & Control & $51(12.3)$ & $365(87.7)$ & & \\
\hline \multirow{4}{*}{ rs2364723 } & & $\mathrm{C}$ & G & \multirow{4}{*}{6.854} & \multirow{4}{*}{0.009} \\
\hline & Case & $112(59.6)$ & $76(40.4)$ & & \\
\hline & Control & $200(48.1)$ & $216(51.9)$ & & \\
\hline & & $\mathrm{C}$ & $\mathrm{T}$ & & \\
\hline \multirow{2}{*}{ rs35652124 } & Case & $109(58.0)$ & $79(42.0)$ & \multirow{2}{*}{5.330} & \multirow{2}{*}{0.021} \\
\hline & Control & $199(47.8)$ & $217(52.2)$ & & \\
\hline \multirow{4}{*}{ rs6721961 } & & $\mathrm{T}$ & G & \multirow{4}{*}{3.682} & \multirow{4}{*}{0.055} \\
\hline & Case & $39(20.7)$ & $149(79.3)$ & & \\
\hline & Control & $117(28.1)$ & 299 (71.9) & & \\
\hline & & $\mathrm{T}$ & $\mathrm{C}$ & & \\
\hline \multirow{2}{*}{ rs2706110 } & Case & $38(20.2)$ & $150(79.8)$ & \multirow{2}{*}{0.949} & \multirow{2}{*}{0.330} \\
\hline & Control & $99(23.8)$ & $317(76.2)$ & & \\
\hline
\end{tabular}

medical histories (hypertension, diabetes, chronic kidney disease, prior aortic stent implant, prior cardiac surgery, smoking, and bicuspid aortic valve) using multivariable linear regression model, we found that rs2364723 (Model 1, $\beta=5.031$, 95\% CI: $1.878-8.183, p=0.002$, Table 6) and rs35652124 (Model 2, $\beta=4.751$, 95\% CI: 1.544-7.958, $p=0.004$, Table 6) were still significantly associated with maximum ascending aorta diameter. Besides, prior cardiac surgery was also found to be significantly associated with maximum ascending aorta diameter (Model 1, $\beta=9.652,95 \% \mathrm{CI}$ : 2.142-17.162, $p=0.012$; Model 2, $\beta=10.228$, 95\% CI: 2.646$17.810, p=0.009$, Table 6). 
TABLE 4: Association of NFE2L2 gene polymorphisms with risk of AAAD.

\begin{tabular}{|c|c|c|c|c|}
\hline SNP & Genetic model & Genotype & OR $(95 \%$ CI $)$ & $p$ value \\
\hline \multirow{3}{*}{ rs1806649 } & Dominant & $(\mathrm{TT}+\mathrm{CT})$ vs. $\mathrm{CC}$ & $1.132(0.605-2.118)$ & 0.699 \\
\hline & Recessive & TT vs. $(\mathrm{CT}+\mathrm{CC})$ & $0.735(0.075-7.158)$ & 1.000 \\
\hline & Homozygote & TT vs. CC & $0.754(0.077-7.370)$ & 1.000 \\
\hline \multirow{3}{*}{ rs13001694 } & Dominant & $(\mathrm{GG}+\mathrm{GA})$ vs. $\mathrm{AA}$ & $1.174(0.667-2.069)$ & 0.578 \\
\hline & Recessive & GG vs. $(\mathrm{GA}+\mathrm{AA})$ & $1.109(0.199-6.161)$ & 1.000 \\
\hline & Homozygote & GG vs. AA & $1.150(0.206-6.425)$ & 1.000 \\
\hline \multirow{3}{*}{ rs2364723 } & Dominant & $(\mathrm{CC}+\mathrm{GC})$ vs. GG & $1.556(0.855-2.829)$ & 0.146 \\
\hline & Recessive & CC vs. $(\mathrm{GC}+\mathrm{GG})$ & $2.069(1.222-3.502)$ & 0.006 \\
\hline & Homozygote & CC vs. GG & $2.333(1.177-4.627)$ & 0.014 \\
\hline \multirow{3}{*}{ rs35652124 } & Dominant & $(\mathrm{CC}+\mathrm{CT})$ vs. TT & $1.490(0.827-2.684)$ & 0.182 \\
\hline & Recessive & CC vs. $(\mathrm{CT}+\mathrm{TT})$ & $1.889(1.112-3.210)$ & 0.018 \\
\hline & Homozygote & CC vs. TT & $2.125(1.076-4.195)$ & 0.029 \\
\hline \multirow{3}{*}{ rs6721961 } & Dominant & $(\mathrm{TT}+\mathrm{GT})$ vs. GG & $0.675(0.411-1.107)$ & 0.118 \\
\hline & Recessive & TT vs. $(\mathrm{GT}+\mathrm{GG})$ & $0.280(0.063-1.249)$ & 0.076 \\
\hline & Homozygote & TT vs. GG & $0.248(0.055-1.123)$ & 0.052 \\
\hline \multirow{3}{*}{ rs2706110 } & Dominant & $(\mathrm{TT}+\mathrm{CT})$ vs. $\mathrm{CC}$ & $0.793(0.481-1.308)$ & 0.364 \\
\hline & Recessive & TT vs. $(\mathrm{CT}+\mathrm{CC})$ & $0.653(0.175-2.429)$ & 0.738 \\
\hline & Homozygote & TT vs. CC & $0.605(0.160-2.282)$ & 0.659 \\
\hline
\end{tabular}

\section{Discussion}

The present study was the first to evaluate the association of NFE2L2 gene polymorphisms with risk and clinical characteristics of AAAD. Our data indicated that the CC genotype of rs2364723 and CC genotype of rs35652124 were risk factors for AAAD. Multivariable linear regression analysis revealed that the CC genotype of rs2364723 and CC genotype of rs35652124 were associated with increased maximum ascending aorta diameter. Besides, patients carrying rs2364723 CC genotype had a higher incidence of coronary artery involvement, while patients carrying rs35652124 CC genotype had a higher incidence of brain ischemia.

The protective role of Nrf2 on aortic dissection and aneurysm has been indicated by several studies. It has been shown that the Nrf2 expression in the thoracic aortic aneurysm tissue from patients with Loeys-Dietz Syndrome was decreased, compared with nondamaged aortic tissue from control subjects [20]. Animal experiments using angiotensin IIinduced aortic aneurysm model in mice showed that NFE2L2 gene deficiency increased the risk of development and rupture of aortic aneurysm [21, 22]. The potential mechanisms underlying the protective role of Nrf2 on aortic dissection and aneurysm may be multifactorial. It has been suggested that activating the Nrf2-ARE pathway suppressed the angiotensin II-induced phenotype switch and proliferation [13], apoptosis [11], calcification [12], and fibrotic [23] of vascular smooth muscle cells. It has also been found that activating the Nrf2-ARE pathway promoted endothelial repair during vascular injury [24].

In the present study, we found that CC genotype of rs2364723 and CC genotype of rs35652124 were linked to an increased risk of AAAD. The rs35652124 polymorphism locates in the promoter region of the NFE2L2 gene, which might regulate Nrf2 expression by influencing the NFE2L2 promoter activity [25]. In vitro transient transfection reporter gene assays showed that rs35652124 C allele reduced the NFE2L2 promoter activity in human microvascular endothelial cells [18]. It has been found that, in healthy African American population, rs35652124 C allele carriers had lower forearm blood flow (FBF) and higher forearm vascular resistance (FVR) under basal condition as well as in response to vasodilators [18]. The maximum FBF response to endotheliumdependent vasodilator in patients with Marfan syndrome has been found to be significantly reduced [26]. These evidences indicate that NFE2L2 gene polymorphisms were associated with vasomotion abnormality, which might play a role in aortic enlargement and dissection. The rs2364723 polymorphism locates in the intron 1 region of the NFE2L2 gene. In a general population-based cohort of Caucasian, it was found that rs2364723 GG carriers had lower cardiovascular mortality and triglyceride levels compared with GC+CC carriers [16]. While abnormal serum lipid composition has been indicated to play a key role in development of aortic dissection [27], it should be noted that the rs2364723 polymorphism was in high linkage disequilibrium (LD) with rs35652124 $\left(r^{2}=0.99\right)$ [28]; thus, the association of rs2364723 with AAAD risk might also represent the effect of rs35652124 due to almost complete LD.

We further investigated the correlation of rs2364723 and rs35652124 polymorphisms with clinical characteristics of AAAD patients and found that rs2364723 CC carriers and rs35652124 CC carriers had increased maximum ascending aorta diameter. Increased ascending aorta diameter is associated with reduced elastin density and increased collagen density, which has a profound impact on aortic dissection [29]. A study on 230 patients with thoracic aortic aneurysm from 
TABLE 5: Association of rs2364723 and rs35652124 polymorphisms with AAAD clinical characteristics.

\begin{tabular}{|c|c|c|c|c|c|c|c|}
\hline \multirow{2}{*}{ Variables } & \multirow{2}{*}{ Overall } & \multicolumn{3}{|c|}{ rs2364723 } & \multicolumn{3}{|c|}{ rs35652124 } \\
\hline & & CC & GC or GG & $p$ & $\mathrm{CC}$ & CT or TT & $p$ \\
\hline Number of patients & 94 & 36 & 58 & & 34 & 60 & \\
\hline \multicolumn{8}{|c|}{ Demographics } \\
\hline Age (years) & $52 \pm 13$ & $52 \pm 13$ & $52 \pm 13$ & 0.865 & $51 \pm 13$ & $52 \pm 13$ & 0.762 \\
\hline Male, $n(\%)$ & $75(80)$ & $30(83)$ & $45(78)$ & 0.500 & $28(82)$ & $47(78)$ & 0.641 \\
\hline \multicolumn{8}{|c|}{ Medical histories, $n(\%)$} \\
\hline Hypertension & $63(67)$ & $25(69)$ & $38(66)$ & 0.694 & $24(71)$ & $39(65)$ & 0.580 \\
\hline Diabetes & $3(3)$ & $2(6)$ & $1(2)$ & 0.556 & $1(3)$ & $2(3)$ & 1.000 \\
\hline CKD & $3(3)$ & $1(3)$ & $2(3)$ & 1.000 & $1(3)$ & $2(3)$ & 1.000 \\
\hline Prior aortic stent implant & $3(3)$ & $1(3)$ & $2(3)$ & 1.000 & $1(3)$ & $2(3)$ & 1.000 \\
\hline Prior cardiac surgery & $5(5)$ & $2(6)$ & $3(5)$ & 1.000 & $1(3)$ & $4(7)$ & 0.650 \\
\hline Smoking & $38(40)$ & $16(44)$ & $22(38)$ & 0.532 & $15(44)$ & $23(38)$ & 0.583 \\
\hline Bicuspid aortic valve & $7(7)$ & $5(14)$ & $2(3)$ & 0.102 & $5(15)$ & $2(3)$ & 0.094 \\
\hline \multicolumn{8}{|c|}{ Clinical presentation } \\
\hline Systolic blood pressure (mmHg) & $151 \pm 27$ & $152 \pm 23$ & $151 \pm 30$ & 0.817 & $151 \pm 22$ & $152 \pm 30$ & 0.870 \\
\hline Diastolic blood pressure (mmHg) & $82 \pm 19$ & $78 \pm 17$ & $84 \pm 20$ & 0.127 & $77 \pm 17$ & $84 \pm 20$ & 0.075 \\
\hline $\mathrm{AoD}_{\text {max }}(\mathrm{mm})$ & $45(41-52)$ & $49(42-57)$ & $42(39-51)$ & 0.002 & $49(42-56)$ & $43(39-52)$ & 0.011 \\
\hline \multicolumn{8}{|c|}{ Organ ischemia, $n(\%)$} \\
\hline Brain & $3(3)$ & $3(8)$ & $0(0)$ & 0.053 & $3(9)$ & $0(0)$ & 0.045 \\
\hline Lower limb & $15(16)$ & $8(22)$ & $7(12)$ & 0.191 & $6(18)$ & $9(15)$ & 0.736 \\
\hline Coronary artery involvement & $18(19)$ & $11(31)$ & $7(12)$ & 0.027 & $10(29)$ & $8(13)$ & 0.057 \\
\hline \multicolumn{8}{|c|}{ Dissection characteristics } \\
\hline \multicolumn{8}{|c|}{ DeBakey classification, $n(\%)$} \\
\hline Type I & $84(89)$ & $32(89)$ & $52(90)$ & \multirow{2}{*}{1.000} & $30(88)$ & $54(90)$ & \multirow{2}{*}{1.000} \\
\hline Type II & $10(11)$ & $4(11)$ & $6(10)$ & & $4(12)$ & $6(10)$ & \\
\hline \multicolumn{8}{|c|}{ Primary entry site, $n(\%)$} \\
\hline Ascending aorta & $62(66)$ & $26(72)$ & $36(62)$ & & $25(74)$ & $37(62)$ & \\
\hline Aortic arch & $19(20)$ & $6(17)$ & $13(22)$ & 0.600 & $5(15)$ & $14(23)$ & 0.490 \\
\hline Descending aorta or unknown & $13(14)$ & $4(11)$ & $9(16)$ & & $4(12)$ & $9(15)$ & \\
\hline \multicolumn{8}{|c|}{ Secondary entry site, $n(\%)$} \\
\hline None & $76(81)$ & $29(81)$ & $47(81)$ & & $28(82)$ & $48(80)$ & \multirow{4}{*}{0.948} \\
\hline Ascending aorta & $2(2)$ & $1(3)$ & $1(2)$ & \multirow{3}{*}{1.000} & $1(3)$ & $1(2)$ & \\
\hline Aortic arch & $14(15)$ & $5(14)$ & $9(16)$ & & $4(12)$ & $10(17)$ & \\
\hline Descending aorta & $2(2)$ & $1(3)$ & $1(2)$ & & $1(3)$ & $1(2)$ & \\
\hline Entry tears $\geq 2, \mathrm{n}(\%)$ & $18(19)$ & $7(19)$ & $11(19)$ & 0.954 & $6(18)$ & $12(20)$ & 0.781 \\
\hline \multicolumn{8}{|c|}{ Aortic valve regurgitation, $n(\%)$} \\
\hline None & $14(15)$ & $4(11)$ & $10(17)$ & \multirow{6}{*}{0.298} & $3(9)$ & $11(18)$ & \multirow{6}{*}{0.205} \\
\hline Mild & $36(38)$ & $14(39)$ & $22(38)$ & & $13(38)$ & $23(38)$ & \\
\hline Mild-moderate & $11(12)$ & $3(8)$ & $8(14)$ & & $3(9)$ & $8(13)$ & \\
\hline Moderate & $14(15)$ & $7(19)$ & $7(12)$ & & $7(21)$ & $7(12)$ & \\
\hline Moderate-severe & $10(11)$ & $2(6)$ & $8(14)$ & & $2(6)$ & $8(13)$ & \\
\hline Severe & $9(10)$ & $6(17)$ & $3(5)$ & & $6(18)$ & $3(5)$ & \\
\hline
\end{tabular}


TABle 5: Continued.

\begin{tabular}{|c|c|c|c|c|c|c|c|}
\hline \multirow{2}{*}{ Variables } & \multirow{2}{*}{ Overall } & \multicolumn{3}{|c|}{ rs2364723 } & \multicolumn{3}{|c|}{ rs35652124 } \\
\hline & & CC & GC or GG & $p$ & $\mathrm{CC}$ & CT or TT & $p$ \\
\hline \multicolumn{8}{|c|}{ Laboratory tests on admission } \\
\hline $\mathrm{WBC}\left(10^{9} / \mathrm{L}\right)$ & $13.2 \pm 4.0$ & $13.1 \pm 4.1$ & $13.3 \pm 4.9$ & 0.904 & $13.1 \pm 4.3$ & $13.3 \pm 3.8$ & 0.878 \\
\hline $\operatorname{PLT}\left(10^{9} / \mathrm{L}\right)$ & $156(124-192)$ & $155(137-184)$ & $157(122-208)$ & 0.800 & $154(137-183)$ & $157(122-211)$ & 0.620 \\
\hline $\mathrm{Hb}(\mathrm{g} / \mathrm{L})$ & $134 \pm 22$ & $133 \pm 24$ & $134 \pm 20$ & 0.957 & $134 \pm 24$ & $133 \pm 20$ & 0.831 \\
\hline $\mathrm{CRP}(\mathrm{mg} / \mathrm{L})$ & $6.8(2.4-20.3)$ & $8.7(2.7-19.2)$ & $5.3(2.3-22.1)$ & 0.602 & $8.7(2.7-21.3)$ & $5.6(2.3-20.9)$ & 0.698 \\
\hline Fibrinogen $(\mathrm{g} / \mathrm{L})$ & $2.13(1.52-2.79)$ & $2.36(1.47-3.03)$ & $1.95(1.52-2.64)$ & 0.621 & $2.31(1.45-3.00)$ & $1.96(1.53-2.74)$ & 0.953 \\
\hline D-dimer ( $\mu \mathrm{g} / \mathrm{mL}$ FEU) & $10.9(4.8-28.6)$ & $8.9(4.2-27.2)$ & $12.0(5.8-29.8)$ & 0.283 & $9.6(4.2-29.9)$ & $11.4(5.7-27.5)$ & 0.544 \\
\hline $\mathrm{UA}(\mu \mathrm{mol} / \mathrm{L})$ & $405 \pm 110$ & $397 \pm 115$ & $411 \pm 107$ & 0.574 & $402 \pm 116$ & $407 \pm 107$ & 0.831 \\
\hline Creatinine $(\mu \mathrm{mol} / \mathrm{L})$ & $98(76-127)$ & $98(78-129)$ & 97 (74-127) & 0.876 & $96(77-124)$ & $98(75-128)$ & 0.642 \\
\hline BUN (mmol/L) & $6.78(5.39-7.98)$ & $6.56(5.36-7.87)$ & $6.91(5.49-8.07)$ & 0.735 & $6.56(5.38-7.68)$ & $6.91(5.41-8.12)$ & 0.645 \\
\hline
\end{tabular}

Data are represented as the median (interquartile range), $n(\%)$, or mean \pm SD. CKD: chronic kidney disease; $A_{0} D_{\max }$ : maximum diameter of ascending aorta; WBC: white blood cell count; PLT: platelet; Hb: hemoglobin; UA: uric acid; BUN: blood urea nitrogen.

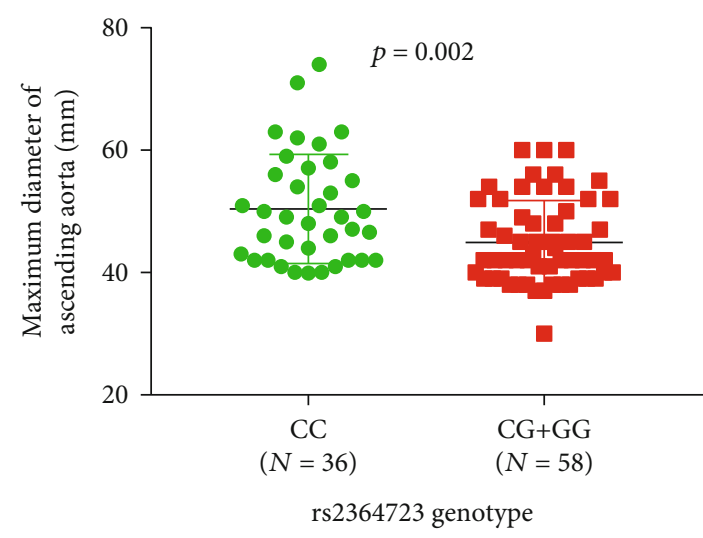

(a)

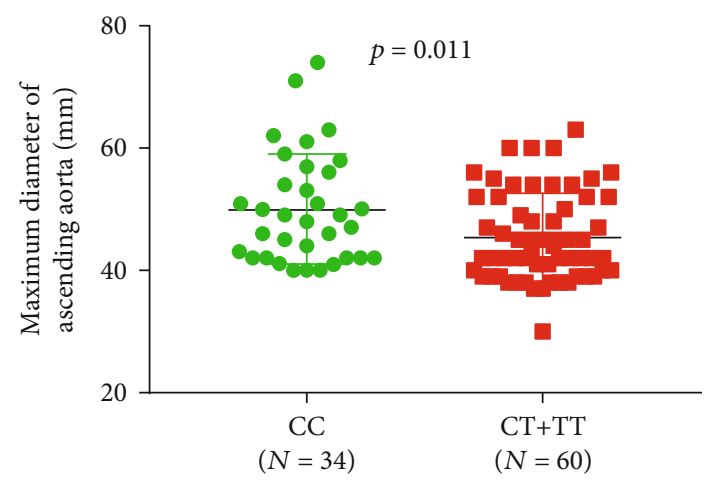

rs35652124 genotype

(b)

Figure 2: Maximum diameter of ascending aorta in AAAD patients grouped by rs2364723 and rs35652124 genotypes.

TABLE 6: Multiple linear regression of maximum ascending aorta diameter.

\begin{tabular}{|c|c|c|c|c|c|c|c|}
\hline Variables & $\begin{array}{l}\text { Model } 1 \\
\quad \beta(95 \% \mathrm{CI})\end{array}$ & $p$ value & Tolerance & Variables & $\begin{array}{l}\text { Model } 2 \\
\quad \beta(95 \% \mathrm{CI})\end{array}$ & $p$ value & Tolerance \\
\hline $\begin{array}{l}\text { rs2364723 } \\
\text { (CC: 1, GC+GG: 0) }\end{array}$ & $5.031(1.878-8.183)$ & 0.002 & 0.943 & $\begin{array}{c}\text { rs35652124 } \\
(\mathrm{CC}: 1, \mathrm{CT}+\mathrm{TT}: 0)\end{array}$ & $4.751(1.544-7.958)$ & 0.004 & 0.946 \\
\hline Age & $0.126(-0.21-0.274)$ & 0.093 & 0.624 & Age & $0.139(-0.10-0.287)$ & 0.068 & 0.625 \\
\hline Male & $0.710(-4.117-5.537)$ & 0.771 & 0.589 & Male & $1.054(-3.806-5.913)$ & 0.667 & 0.590 \\
\hline Hypertension & $-2.945(-6.213-0.323)$ & 0.077 & 0.938 & Hypertension & $-3.149(-6.447-0.149)$ & 0.061 & 0.935 \\
\hline Diabetes & $2.338(-6.946-11.622)$ & 0.618 & 0.831 & Diabetes & $4.093(-5.196-13.382)$ & 0.383 & 0.843 \\
\hline CKD & $1.383(-7.946-10.712)$ & 0.769 & 0.823 & CKD & $0.632(-8.762-10.027)$ & 0.894 & 0.824 \\
\hline $\begin{array}{l}\text { Prior aortic stent } \\
\text { implant }\end{array}$ & $6.274(-2.376-14.925)$ & 0.153 & 0.957 & $\begin{array}{l}\text { Prior aortic stent } \\
\text { implant }\end{array}$ & $6.379(-2.337-15.096)$ & 0.149 & 0.957 \\
\hline Prior cardiac surgery & $9.652(2.142-17.162)$ & 0.012 & 0.779 & Prior cardiac surgery & $10.228(2.646-17.810)$ & 0.009 & 0.776 \\
\hline Smoking & $-0.497(-3.929-2.934)$ & 0.774 & 0.780 & Smoking & $-0.553(-3.992-2.926)$ & 0.760 & 0.780 \\
\hline Bicuspid aortic valve & $4.633(-1.274-10.540)$ & 0.123 & 0.920 & Bicuspid aortic valve & $4.620(-1.348-10.588)$ & 0.127 & 0.915 \\
\hline
\end{tabular}

CKD: chronic kidney disease.

1985 to 1996 showed that the median diameter of ascending aorta was $6.0 \mathrm{~cm}$ at time of aorta rupture or dissection [30]. More recent data on nature history of ascending aortic aneu- rysm between 5 and $6 \mathrm{~cm}$ found two hinge points for aorta rupture or dissection-one at $5.25 \mathrm{~cm}$ and the other at $5.75 \mathrm{~cm}$ [31]. Recent studies also indicated that aortic 
diameter $\geq 40 \mathrm{~mm}$ was an independent risk factor for inhospital mortality of acute aortic dissection [32], and increased ascending aortic diameter was found to be negatively correlated with total survival time in patients with ascending aortic dissection [33]. Our study also found the correlation of rs2364723 and rs35652124 polymorphisms with incidence of organ ischemia such as brain ischemia and coronary artery involvement. Recent data showed that vital organ ischemia was a risk factor for early mortality after AAAD repair [34]. These evidences suggested that NFE2L2 gene polymorphisms not only correlated with onset risk but also severity of AAAD.

There are several limitations in the present study. Firstly, it is a pilot, single-center research with relatively small sample size, and our findings cannot be generalized to the general population since we included only Han Chinese subjects. Secondly, this study only focused on 6 SNPs previously reported to be associated with cardiovascular risk, while other SNPs with potential influence were not examined. Moreover, we had not collected the aortic tissue of the AAAD patients, which made us unable to measure the oxidative stress condition in dissected aorta of the patients. Thus, further multicenter studies with different ethnicities and larger sample size were needed, and additional studies should determine the mechanisms underlying the effects of gene polymorphisms on functions of NFE2L2, and its role in the pathogenesis of AAAD.

\section{Conclusions}

The present study showed that rs2364723 and rs35652124 polymorphisms in NFE2L2 gene were correlated with risk of AAAD in a Han Chinese population. These two SNPs were also related to maximum ascending aorta diameter and vital organ ischemia in AAAD patients.

\section{Data Availability}

The data used to support the findings of this study are available from the corresponding authors upon request.

\section{Conflicts of Interest}

The authors declare that there is no conflict of interest regarding the publication of this paper.

\section{Acknowledgments}

This work was supported by grant from the Natural Science Foundation of Zhejiang Province of China (Grant No. LQ20H020003) and the Key Research and Development Program of Zhejiang Province, China (Grant No. 2019C03008).

\section{References}

[1] D. P. Howard, A. Banerjee, J. F. Fairhead et al., "Populationbased study of incidence and outcome of acute aortic dissection and premorbid risk factor control: 10-year results from the Oxford Vascular Study," Circulation, vol. 127, no. 20, pp. 2031-2037, 2013.
[2] R. H. Mehta, T. Suzuki, P. G. Hagan et al., "Predicting death in patients with acute type A aortic dissection," Circulation, vol. 105, no. 2, pp. 200-206, 2002.

[3] E. M. Isselbacher, C. L. Lino Cardenas, and M. E. Lindsay, "Hereditary influence in thoracic aortic aneurysm and dissection," Circulation, vol. 133, no. 24, pp. 2516-2528, 2016.

[4] T. Li, X. Liu, H. Ning, X. Li, J. Yang, and C. Ma, "Association of Toll-like receptor 4 gene polymorphisms with acute aortic dissection in a Chinese Han population," BioMed Research International, vol. 2020, Article ID 8306903, 11 pages, 2020.

[5] K. Yang, J. Ren, X. Li et al., "Prevention of aortic dissection and aneurysm via an ALDH2-mediated switch in vascular smooth muscle cell phenotype," European Heart Journal, vol. 41, no. 26, pp. 2442-2453, 2020.

[6] J. Han, J. Liu, Q. Zhou, S. Nie, J. Liu, and S. Wen, "Single nucleotide polymorphisms (SNPs) genotyping reveals that Mfn2 polymorphisms are associated with thoracic aortic dissection in Han Chinese population," Medical Science Monitor, vol. 25, pp. 2419-2428, 2019.

[7] M. Liao, Z. Liu, J. Bao et al., "A proteomic study of the aortic media in human thoracic aortic dissection: implication for oxidative stress," The Journal of Thoracic and Cardiovascular Surgery, vol. 136, no. 1, pp. 65-72.e3, 2008, e1-3.

[8] L. M. Fan, G. Douglas, J. K. Bendall et al., "Endothelial cellspecific reactive oxygen species production increases susceptibility to aortic dissection," Circulation, vol. 129, no. 25, pp. 2661-2672, 2014.

[9] L. Xia, C. Sun, H. Zhu et al., "Melatonin protects against thoracic aortic aneurysm and dissection through SIRT1dependent regulation of oxidative stress and vascular smooth muscle cell loss," Journal of Pineal Research, vol. 69, no. 1, p. e12661, 2020.

[10] P. Shaw and A. Chattopadhyay, "Nrf2-ARE signaling in cellular protection: mechanism of action and the regulatory mechanisms," Journal of Cellular Physiology, vol. 235, no. 4, pp. 3119-3130, 2020.

[11] T. Xiao, L. Zhang, Y. Huang et al., "Sestrin2 increases in aortas and plasma from aortic dissection patients and alleviates angiotensin II-induced smooth muscle cell apoptosis via the Nrf2 pathway," Life Sciences, vol. 218, pp. 132-138, 2019.

[12] R. Wei, M. Enaka, and Y. Muragaki, "Activation of KEAP1/NRF2/P62 signaling alleviates high phosphate-induced calcification of vascular smooth muscle cells by suppressing reactive oxygen species production," Scientific Reports, vol. 9, no. 1, p. 10366, 2019.

[13] X. He, J. Deng, X. J. Yu, S. Yang, Y. Yang, and W. J. Zang, "Activation of M3AChR (type 3 muscarinic acetylcholine receptor) and Nrf2 (nuclear factor erythroid 2-related factor 2) signaling by choline alleviates vascular smooth muscle cell phenotypic switching and vascular remodeling," Arteriosclerosis, Thrombosis, and Vascular Biology, vol. 40, no. 11, pp. 2649-2664, 2020.

[14] I. Sarutipaiboon, N. Settasatian, N. Komanasin et al., "Association of genetic variations in NRF2, NQO1, HMOX1, and MT with severity of coronary artery disease and related risk factors," Cardiovascular Toxicology, vol. 20, no. 2, pp. 176-189, 2020.

[15] S. Shimizu, J. Mimura, T. Hasegawa et al., "Association of single nucleotide polymorphisms in the NRF2 promoter with vascular stiffness with aging," PLoS One, vol. 15, no. 8, article e0236834, 2020.

[16] S. M. Figarska, J. M. Vonk, and H. M. Boezen, "NFE2L2 polymorphisms, mortality, and metabolism in the general 
population," Physiological Genomics, vol. 46, no. 12, pp. 411417, 2014.

[17] B. Wang, M. Liu, W. Yan et al., "Association of SNPs in genes involved in folate metabolism with the risk of congenital heart disease," The Journal of Maternal-Fetal \& Neonatal Medicine, vol. 26, no. 18, pp. 1768-1777, 2013.

[18] E. D. Marczak, J. Marzec, D. C. Zeldin et al., "Polymorphisms in the transcription factor NRF2 and forearm vasodilator responses in humans," Pharmacogenetics and Genomics, vol. 22, no. 8, pp. 620-628, 2012.

[19] T. Kunnas, K. Maatta, and S. T. Nikkari, "Genetic polymorphisms of transcription factor NRF2 and of its host gene sulfiredoxin (SRXN1) are associated with cerebrovascular disease in a Finnish cohort, the TAMRISK study," International Journal of Medical Sciences, vol. 13, no. 5, pp. 325-329, 2016.

[20] M. E. Soto, L. G. Manzano-Pech, V. Guarner-Lans et al., "Oxidant/antioxidant profile in the thoracic aneurysm of patients with the Loeys-Dietz syndrome," Oxidative Medicine and Cellular Longevity, vol. 2020, Article ID 5392454, 17 pages, 2020.

[21] A. Kopacz, E. Werner, A. Grochot-Przeczek et al., "Simvastatin attenuates abdominal aortic aneurysm formation favoured by lack of Nrf2 transcriptional activity," Oxidative Medicine and Cellular Longevity, vol. 2020, Article ID 6340190, 16 pages, 2020.

[22] H. Song, T. Xu, X. Feng et al., "Itaconate prevents abdominal aortic aneurysm formation through inhibiting inflammation via activation of Nrf2," eBioMedicine, vol. 57, p. 102832, 2020.

[23] Y. C. Hseu, T. Y. Yang, M. L. Li et al., "Chalcone flavokawain A attenuates TGF- $\beta 1$-induced fibrotic pathology via inhibition of ROS/Smad3 signaling pathways and induction of Nrf2/ARE-mediated antioxidant genes in vascular smooth muscle cells," Journal of Cellular and Molecular Medicine, vol. 23, no. 2, pp. 775-788, 2019.

[24] J. Zhang, W. Cai, Z. Fan et al., "MicroRNA-24 inhibits the oxidative stress induced by vascular injury by activating the Nrf2/Ho-1 signaling pathway," Atherosclerosis, vol. 290, pp. 9-18, 2019.

[25] J. M. Marzec, J. D. Christie, S. P. Reddy et al., "Functional polymorphisms in the transcription factor NRF2 in humans increase the risk of acute lung injury," The FASEB Journal, vol. 21, no. 9, pp. 2237-2246, 2007.

[26] M. Nakamura, S. Itoh, S. Makita, A. Ohira, N. Arakawa, and K. Hiramori, "Peripheral resistance vessel dysfunction in Marfan syndrome," American Heart Journal, vol. 139, no. 4, pp. 661-666, 2000.

[27] H. Tanaka, Y. Iida, T. Iwaki et al., "Elevated plasma levels of LDL cholesterol promote dissecting thoracic aortic aneurysms in angiotensin II-induced mice," Annals of Vascular Surgery, vol. 48, pp. 204-213, 2018.

[28] M. Siedlinski, D. S. Postma, J. M. Boer et al., "Level and course of FEV1 in relation to polymorphisms in NFE2L2 and KEAP1 in the general population," Respiratory Research, vol. 10, no. 1, 2009.

[29] V. Deplano, M. Boufi, V. Gariboldi et al., "Mechanical characterisation of human ascending aorta dissection," Journal of Biomechanics, vol. 94, pp. 138-146, 2019.

[30] M. A. Coady, J. A. Rizzo, G. L. Hammond et al., "What is the appropriate size criterion for resection of thoracic aortic aneurysms?," The Journal of Thoracic and Cardiovascular Surgery, vol. 113, no. 3, pp. 476-491, 1997, discussion 89-91.
[31] B. A. Ziganshin, M. A. Zafar, and J. A. Elefteriades, "Descending threshold for ascending aortic aneurysmectomy: is it time for a "left-shift" in guidelines?," The Journal of Thoracic and Cardiovascular Surgery, vol. 157, no. 1, pp. 37-42, 2019.

[32] D. Wen, P. Jia, X. Du, J. Z. Dong, and C. S. Ma, "Value of $\mathrm{N}$-terminal pro-brain natriuretic peptide and aortic diameter in predicting in-hospital mortality in acute aortic dissection," Cytokine, vol. 119, pp. 90-94, 2019.

[33] Y. Wu, M. Gong, R. Fan, T. Gu, X. Qian, and H. Zhang, “Analysis of ascending aortic diameter and long-term prognosis in patients with ascending aortic dissection," Echocardiography, vol. 38, no. 4, pp. 531-539, 2021.

[34] M. Yamasaki, H. Yoshino, T. Kunihara et al., "Risk analysis for early mortality in emergency acute type A aortic dissection surgery: experience of Tokyo acute aortic super-network," European Journal of Cardio-Thoracic Surgery, 2021. 